\title{
Therapeutische Aspekte zur Hilfsmittelversorgung von Patienten mit Multipler Sklerose
}

Neurol Rehabil 2020; 26(3): 179-184 (C) Hippocampus Verlag 2020

DOI $10.14624 / \mathrm{NR} 2008006$

\author{
S. Lamprecht ${ }^{1}$, T. Böing ${ }^{2}$
}

\section{Zusammenfassung}

Hilfsmittel können wesentlich zur Eigenaktivität und Teilhabe von Patienten mit Multipler Sklerose (PwMS) beitragen. Sie sollten sorgfältig ausgewählt und angepasst sowie gegebenenfalls im Krankheitsverlauf modifiziert werden. Der Beitrag stellt verschiedene Hilfsmittel vor und erläutert deren Verordnung und Einsatz im häuslichen Bereich.

Schlüsselwörter: Hilfsmittel, Orthesen, funktionelle Elektrostimulation, Rollstuhl, Trainingsempfehlung

\footnotetext{
${ }^{1}$ Praxis Lamprecht, HSH Lamprecht GbR, Kirchheim/Teck

${ }^{2}$ Otto Bock HealthCare Deutschland, Duderstadt
}

\section{Einführung}

Als häufigste Autoimmunerkrankung des zentralen Nervensystems (ZNS) kommt es bei Multipler Sklerose zu einer fortschreitenden Schädigung der Myelinhüllen infolge eines chronisch-entzündlichen Prozesses. Aktuell erkranken in Deutschland jedes Jahr etwa 12.600 neue Patienten an Multipler Sklerose, die Gesamthäufigkeit liegt bei ca. 224.000 Patienten [13]. Frauen sind deutlich häufiger von MS betroffen als Männer [8], wobei eine exakte Bestimmung von Ursachen und Verlaufsformen bis heute nicht eindeutig ist [34]. Während die demographische Entwicklung im Bereich der DRV zu einem geringen Rückgang der Fallzahlen führen wird
(-5,2\%), ist für den Bereich der GKV ein deutlicher Anstieg zu erwarten (25,9\%) [21]. Der wachsende Anteil von Patienten mit Multipler Sklerose (PwMS) bei gleichzeitig signifikanter Steigerung der »Disability-Adjusted Life-Years« [9] führt nicht nur zu einer erhöhten Therapienachfrage, sondern ebenfalls - in Abhängigkeit vom Krankheitsgrad überproportional zunehmend - zu einer Zunahme gesundheitsökonomischer Belastungen [7]. Ungeachtet dessen sind die Ausgaben für Heil- und Hilfsmittel im Vergleich zu anderen Leistungsbereichen der GKV marginal. So betrugen sie 2018 gerade einmal 3,73\% und 3,35\% und stellen nach wie vor keineswegs einen primär kostentreibenden Faktor im Gesundheitswesen dar (Abb. 1) [10a].

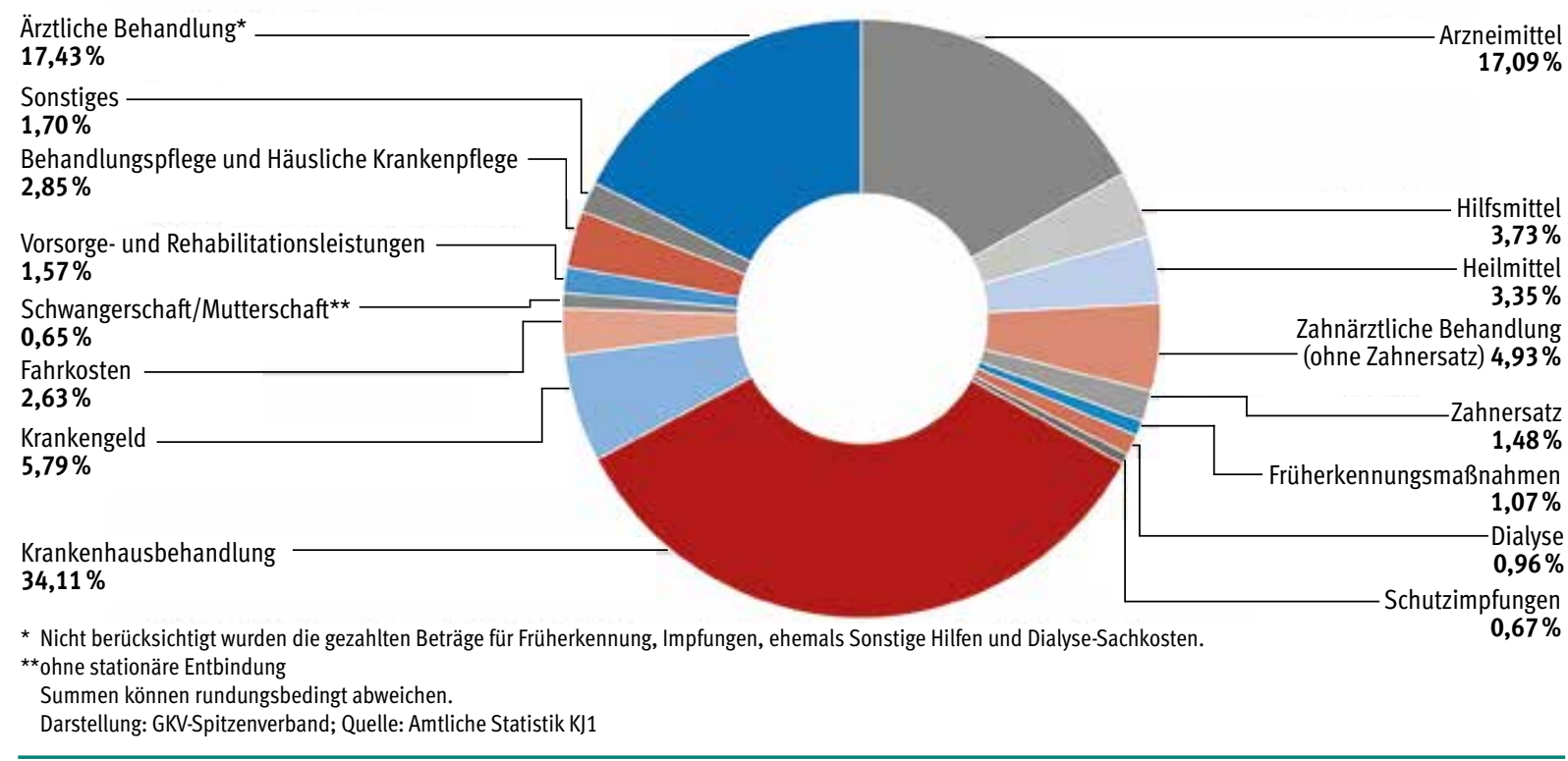

Abb. 1: Ausgaben für einzelne Leistungsbereiche der GKV 2018 in Prozent 
Therapeutic aspects with regard to the supply of medical devices to patients with multiple sclerosis

S. Lamprecht, T. Böing

\section{Abstract}

Medical devices can make a significant contribution to the self-activity and participation of patients with multiple sclerosis (PwMS). They should be selected and adapted carefully and, if necessary, modified as the disease progresses. The article presents various medical devices and explains their prescription and use in everyday life.

Keywords: aids, orthoses, functional electrical stimulation, wheelchair, training recommendation

Neurol Rehabil 2020; 26(3): 179-184, DOI 10.14624/NR2008006

(C) Hippocampus Verlag 2020
Neben dem SGB V ist die Hilfsmittelversorgung für PwMS im »Rahmenvertrag Entlassmanagement« (§39 Abs. 1a S. 9 SGB V) definiert. Die Vertragspartner sind der GKV-Spitzenverband, die Kassenärztliche Bundesvereinigung und die Deutsche Krankenhausgesellschaft [10]. Dieser Vertrag regelt u.a. den Übergang stationärambulant und beinhaltet den wichtigen Passus, dass Hilfsmittel dauerhaft, also auch für die Zeit nach der Krankenhausbehandlung, vom Klinikarzt auf dem Formular »Entlassrezept« verordnet werden können. Welches Hilfsmittel erforderlich ist, entscheidet im Idealfall einer multidisziplinäres Therapieteam. Der zuständige Arzt verordnet es im Rahmen seiner Therapiefreiheit, das Vorgehen ist in der Hilfsmittelrichtlinie des Gemeinsamen Bundesausschusses (G-BA) geregelt [23]. Die Entscheidung muss keineswegs ausführlich schriftlich begründet werden, eine kurze medizinische Begründung auf dem Rezept ist jedoch empfehlenswert, denn es unterstützt die Verordnungstransparenz und erleichtert den Krankenkassen den Arbeitsprozess.

\section{Therapie- und Trainingsempfehlungen}

Ein aktuelles Cochrane-Review unterstreicht einmal mehr die Notwendigkeit strukturierter und multidisziplinärer Rehabilitationsprogramme für PwMS. Mit gezielten Inhalten zu Bewegung und körperlicher Aktivität lassen sich

- Mobilität,

- Muskelkraft,

- aerobe Kapazität und die

- Lebensqualität nachweislich verbessern [1].

Die passenden Trainingsformen mit den jeweiligen mobilitätsbezogenen Outcomes finden sich im Detail in der aktuellen S2e-Leitlinie der DGNR in Zusammenarbeit mit Physio Deutschland [32]. Die wichtigsten Empfehlungen sind:

- Vorrangig sollte ein regelmäßiges, therapeutisch angeleitetes Gangtraining stattfinden.

- Unterstützend sollte ein systematisches Ausdauertraining mittels Laufband, Ergometer, Gehen und geziel- tes Krafttraining für Fußheber, Hüftbeuger, Wadenmuskulatur und Quadriceps durchgeführt werden.

- Für schwer Betroffene (EDSS > 6) sollte roboterassistiertes Gangtraining eingesetzt werden.

- Regelmäßige Physiotherapie sollte immer funktionell, ziel- und alltagsorientiert auf die bestehenden Beeinträchtigungen ausgerichtet sein (ICF: International Classification of Functioning, Disability and Health).

Individualisierte Trainingsprogramme verbessern nachweislich Fitness, Funktionsfähigkeit und Lebensqualität [12], und eine aktuelle Untersuchung konkretisiert die Trainingsrichtlinien für PwMS wie folgt:

- 2-3 mal pro Woche aerobes Training (10-30 Minuten bei mittlerer Intensität).

- 2-3 mal pro Woche Kraft- bzw. Widerstandstraining (1-3 Sets zwischen 8 und 15 Wiederholungsmaxima) [18].

Für den Alltag können sich Dauer und Intensität körperlicher Aktivität ebenso an den »Nationalen Bewegungsempfehlungen « orientieren, immer unter Berücksichtigung der patientenspezifischen Beeinträchtigungen [24]. Konsequent umgesetzt, führt die Kombination verschiedener Therapie- und Trainingsinhalte zu Verbesserungen von Gleichgewicht, Muskelkraft, Beweglichkeit und Ausdauer [25, 31]. Für Patienten, die für das therapeutische Klettern in Betracht kommen, zeigt sich neben den Inhalten des motorischen Lernens zudem weitere positive Aspekte, nämlich:

- Verbesserung von kognitiven und psychischen Funktionseinschränkungen sowie

- ausgeprägte starke soziale und gruppendynamische Komponenten [14].

Dennoch gibt es nach wie vor eine große Anzahl von PwMS, die Alltagstraining und Bewegung meiden. Als Gründe gelten primär der fehlende Transfer von Forschungsergebnissen in die Praxis, insbesondere aber auch ein gezielter Schulungsbedarf für Patienten und Therapeuten [15].

\section{Therapieunterstützung durch Hilfsmittel}

Wie in vielen anderen Bereichen der Medizin gibt es inzwischen zahlreiche innovative Verbesserungen. So stellt beispielsweise die funktionelle Elektrostimulation (FES) eine inzwischen mehr als etablierte Versorgungsoption für die Fußheberschwäche dar, sehr häufig auftretend bei PwMS . Zeitgemäße FES-Systeme verbinden neueste medizinisch-technische Errungenschaften mit therapeutischem Know-how und bieten in der Versorgung von PwMS besondere Perspektiven. Sie werden direkt am Körper getragen, können je nach Bedarf vom Patienten und Therapeuten ein- oder ausgeschaltet wer- 


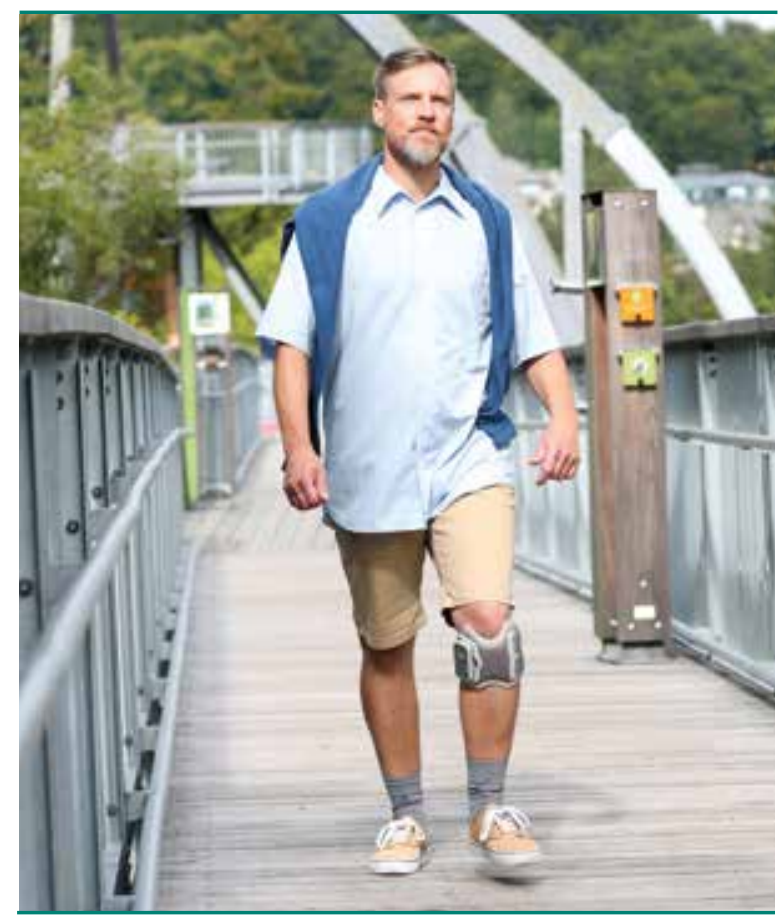

Abb. 2: FES-System im Alltag

den und sind sowohl für die stationäre und ambulante Rehabilitation als auch für den alltäglichen Einsatz im Privatbereich geeignet (Abb. 2). Durch eine echtzeitgenaue Gangerkennung wird unmittelbar vor der Schwungphase ein Stimulationsimpuls gesendet, der einen sicheren und aktiven Bewegungsablauf gewährleistet und das Gehen weitestgehend normalisieren kann. So konnte bei PwMS eine signifikante Reduktion der wahrgenommenen Anstrengung beim Gehen sowie eine deutliche Verbesserung der Lebensqualität nachgewiesen werden $[17,28,29]$. Insbesondere unter dem ICF-Aspekt der Teilhabe zeigen diese Versorgungsoptionen einen deutlichen Mehrwert im Vergleich zum bewährten Versorgungsstandard mit Orthesen, bedingen jedoch von Anfang an eine präzise Patientenselektion [3]. Folgende Gütekriterien sollte ein sicheres FES-System vorweisen:

- Ausbalancierte Dorsalextension und Eversion

- Separate Ansteuerung von N. peronaeus communis und M. tibialis anterior für eine saubere Fußhebung

- Absolut zuverlässige 3D-Bewegungsdetektion mittels Gyroskop und Akzelerometer

- Einfache Repositionierung und kurze Rüstzeit

- Möglichkeit, durch einfache Impulsverstärkung auf die motorische Fatigue einzugehen

- Trainingsmodus für aktives und gezieltes Training des Fußhebers

- Integrierte Assessments (z.B. 10m-Gehtest) zur smarten Dokumentation

- Upgrade-Option für ein Oberschenkelmodul zur Vermeidung einer Hyperextension im Kniegelenk (z. B. bei Voranschreiten des Krankheitsbildes) (Abb. 3).

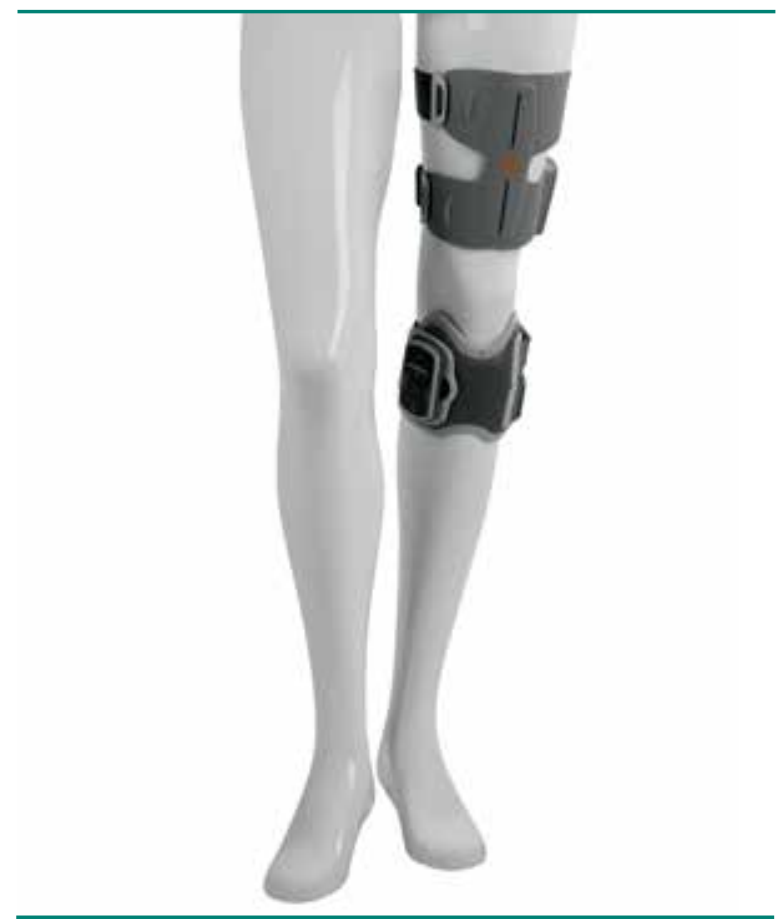

Abb. 3: FES-System mit Oberschenkelmodul

Da funktionell gesehen PwMS besonders häufig Probleme mit der Schwungphase haben, profitieren diese in besonderem Maße von FES, da die funktionelle Elektrostimulation hier und zu Beginn der Standphase (Initial Contact, Loading Response) besonders effektiv unterstützen kann. Die Problematik der insuffizienten Hüftflexoren, die sich ebenfalls in der Spielbeinphase bemerkbar macht (das Bein kann nur schwer nach vorne gebracht werden), kann durch die FES ebenfalls positiv beeinflusst werden. Hier hilft die FES indirekt, indem sie den Fußheber unterstützt und damit der ohnehin schwache Hüftbeuger den Fallfuß nicht mehr kompensieren muss.

Neben der funktionellen Elektrostimulation können Orthesen ebenfalls einen signifikanten Beitrag zur Verbesserung von Gangsicherheit und Lebensqualität leisten [27]. Allerdings sollten diese möglichst leicht sein, da zu schwere Orthesen als distales Gewicht bei PwMS mit schwachen Hüftflexoren kontraproduktiv wirken und es bei falscher Auswahl des Hilfsmittels somit zu einer funktionellen Verschlechterung des Gangbildes kommen kann. Patienten empfinden dann ein deutlich schwereres Bein und akzeptieren folglich die Orthese häufig nicht. Eine umfangreiche Befragung betroffener Patienten zeigte auch, dass genauere Informationen $\mathrm{zu}$ Gebrauch und Handhabung von Orthesen sowie Verbesserungen in der Ästhetik wünschenswert wären [30]. Orthesen mit eingebauter motorisierter Unterstützung von Dorsal- und Plantarflexion (PRAFO, Passive or Powered Ankle-Foot Orthoses) liefern keinerlei Mehrwert [2]. Knieübergreifende Orthesenversorgungen (KAFO = Knee Ankle Foot Orthoses) kommen zum Einsatz, wenn 


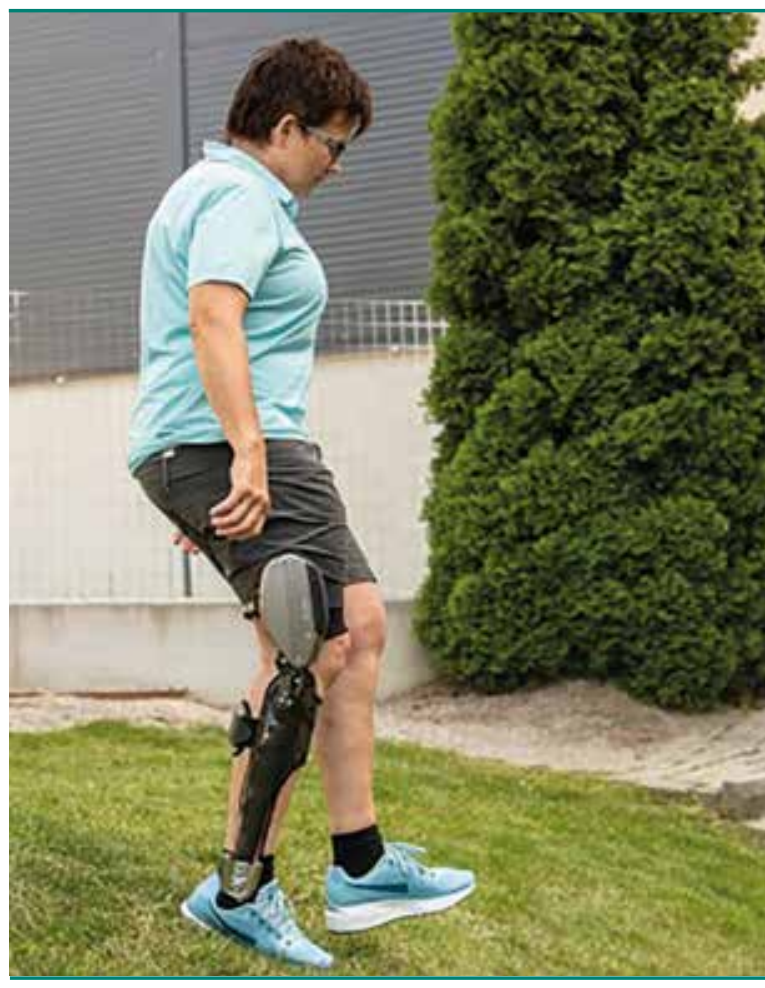

Abb. 4: ADL mit SSCO

mehrere große Muskelgruppen betroffen sind. Mit einer durchgängigen Sperrung des Kniegelenks erreicht man zwar das größtmögliche Maß an Gangsicherheit in der Standphase, diese ist jedoch mit einer Mehrbelastung des Bewegungsapparats und des Energieverbrauchs verknüpft $[20,26]$. Eine Stance Control Orthosis (SCO) kann diese Nachteile nachweislich signifikant reduzieren [33]. Hier sorgt ein Umschaltmechanismus für die Sperrung des Kniegelenks unter Belastung und die Freigabe für die Schwungphase, sicheres Gehen in der Ebene ist somit möglich. Funktionell limitierend ist bei diesen Orthesen jedoch, dass keine gedämpfte Knieflexion unter Belastung gegeben ist. Das hingegen bieten »Stance and Swing Phase Controlled Orthoses« (SSCO): mit diesen Orthesen können auch Treppen und Schrägen im Alltag bewältigt werden, denn eine mikroprozessorgesteuerte Hydraulikeinheit garantiert für alle Bewegungsabläufe des Alltags optimierte Bewegungswiderstände bzgl. Flexion und Extension des Kniegelenkes (Abb. 4). Wie zuvor bereits beschrieben, darf das Gewicht dieser Orthesen das Gehen nicht beschwerlicher machen, auch ist der funktionelle Zugewinn der mikroprozessorgesteuerten Systeme bis dato speziell bei PwMS methodisch nicht hochgradig belegt. Vorteile ergeben sich jedoch sicherlich bei Patienten mit deutlich insuffizientem $M$. quadriceps bei gleichzeitig suffizienten Hüftflexoren. So lässt sich festhalten, dass insbesondere Patienten mit Rückenmarksverletzungen, N. femoralis-Läsionen oder Patienten mit einem Ausfall des M. quadriceps profitieren. Diese Systeme können sich auf variierende Geh-

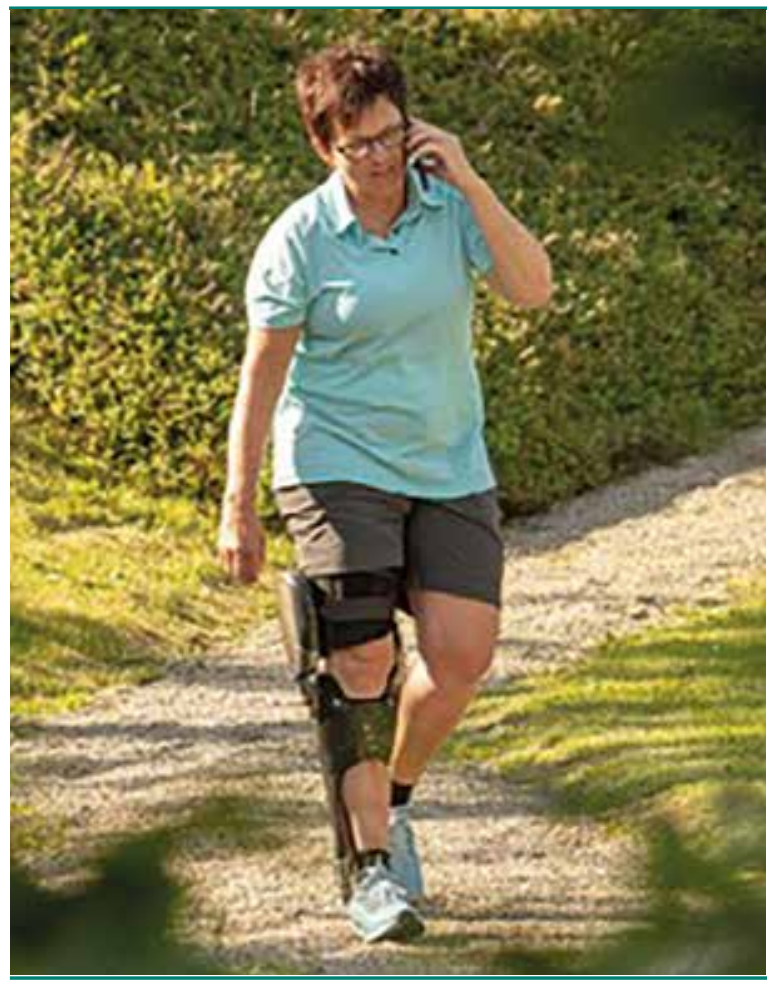

Abb. 5: Dual Task mit SSCO

geschwindigkeiten und individuelle Kniebewegungen einstellen. Größter Patientennutzen dieser Systeme ist jedoch das extrem hohe Sicherheitspotential, das selbst Dual-Task-Aktivität im Alltag wieder ermöglicht (Abb. 5). Selbst bei einem Stolpern wird der Sturz vermieden, denn die sensorbasierte Steuerung reagiert in Echtzeit, d.h. der vollständige Flexionswiderstand greift sofort, der Patient stürzt nicht.

Zwischenergebnisse einer randomisierten kontrollierten Studie zeigen, dass stand- und schwungphasengesteuerte Orthesen im Vergleich zu gesperrten (KAFO) oder allein schwungphasengesteuerten Orthesen (SCO) deutlich überlegen sind. So zeigen sich $85 \%$ (im Vergleich zur SCO) bzw. 65\% (im Vergleich zur KAFO) weniger Stürze, zudem signifikante Verbesserungen bei

- der Gehgeschwindigkeit,

- der Gangqualität,

- dem 6-Minuten-Gehtest sowie

- auf Stufen, Rampen und unebenem Gelände.

Ob sich diese Ergebnisse bei PwMS auch darstellen lassen, bleibt abzuwarten. Wichtig ist eine umfassende Erprobung. Sollte der Hüftflexor sehr schwach sein, kann der oben beschriebene Vorteil dieser schwungphasengesteuerten Orthesen aufgehoben werden und eher zu negativen Ergebnissen führen.

Zusammengefasst kann das im Einzelfall für PwMS, die mit einer SSCO versorgt sind, bedeuten

- mehr Sicherheit,

- mehr ADL-relevante Funktionen 
mehr Mobilität [4] sowie

n ein signifikanter Mehrwert in nahezu allen ADL-relevanten Items zum Patient Reported Outcome (PRO) [22].

Dennoch muss - analog zur Vorgehensweise bei der FES - für die Versorgung mit einer SSCO ein individuelles Patientenscreening vorausgehen, das u.a. mithilfe einer eindeutigen Video-Dokumentation (Treppe, Ebene, Rampe) den Mehrwert für den zu versorgenden Patienten zeigt. Auch der Bedarf muss immer kritisch geprüft werden: Ist wirklich der Ausfall des M. quadriceps das größte funktionelle Problem des Patienten, oder spielt die Schwäche der Hüftflexoren im Gesamtkontext doch die bedeutendere Rolle?

Sind Orthesen oder FES-Systeme nicht mehr ausreichend, steht häufig eine Rollstuhlversorgung an. Gemäß der Deutschen Vereinigung für Rehabilitation e. V. gelten dabei folgende Gütekriterien:

- adäquate Produktwahl unter Berücksichtigung der individuellen Teilhabeziele und Kontextfaktoren (ICF),

- alltagsorientiertes Training in der Anwendung,

- ausreichende Unterweisung in den Gebrauch,

- Anpassung und ggf. die individuelle Gestaltung des Sitzes,

- Beratungsqualität,

zeitnah erreichbarer Service.

Dabei kommt laut DVfR dem »Grad der Selbstständigkeit bei der Mobilität eine hohe Bedeutung für die praktische Realisierung der Selbstbestimmung bei der Teilhabe zu, so dass auch das Antriebssystem (z.B. sog. Schieberollstuhl, Aktivrollstuhl, elektrisch angetriebener Rollstuhl) einen wichtigen Aspekt der Rollstuhlversorgung darstellt« [6]. Eine Versorgungsoption ist in Abbildung 6 dargestellt.

\section{Zusammenfassung}

Zeitgemäße Therapieansätze fordern und fördern die Eigenaktivität von PwMS. Dieses Empowerment transportiert nicht allein körperliche Coping-Stragegien. Falls notwendig, sollten dabei Hilfsmittel unterstützend und ADL-vorbereitend in die Therapie implementiert werden. Sie bedürfen immer einer patientenspezifischen Selektion und Anpassung und unterliegen bei fortschreitendem Krankheitsverlauf u.U. einer Versorgungsmodifikation. Gütekriterien, an denen sich die Versorgungsoptionen messen lassen können, sind u. a. Compliance und Hilfsmitteladhärenz. Letztendlich sollte das Hilfsmittel seitens der PwMS auch adäquat genutzt werden, um Aktivität und Teilhabe so lange wie möglich zu gewährleisten.

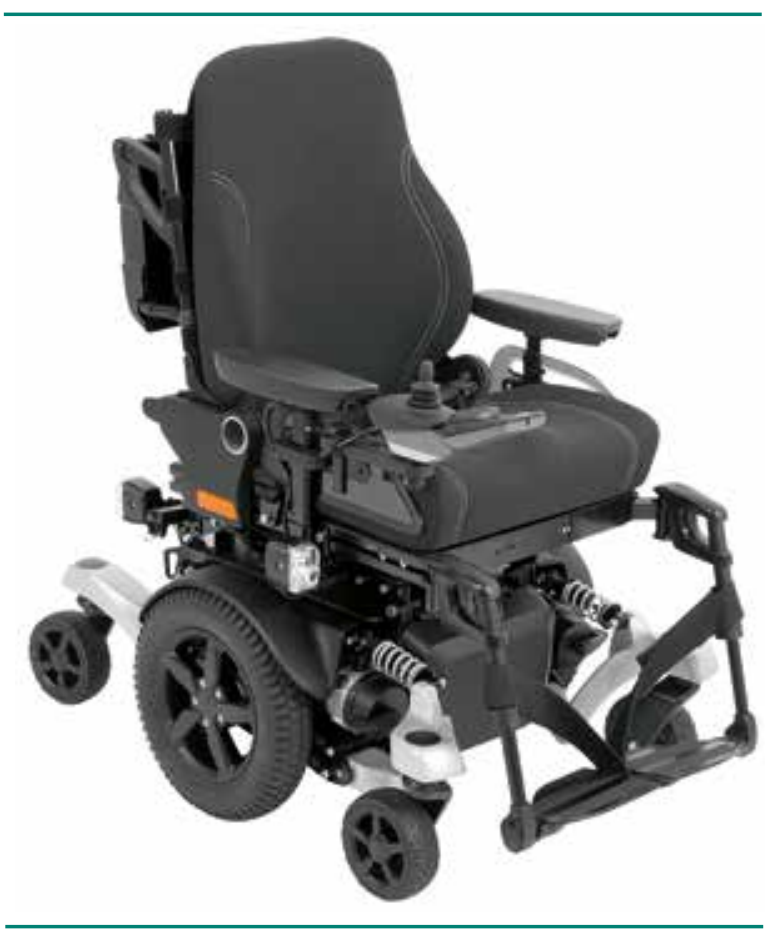

Abb. 6: E-Rollstuhl

\section{Literatur}

1. Amatya B, Khan F, Galea M. Rehabilitation for people with multiple sclerosis: an overview of Cochrane Reviews. Cochrane Database Syst Rev 2019; doi:10.1002/14651858.CDo12732.pub2.

2. Boes MK, Bollaert RE, Kesler RM, Learmonth YC, Islam M, Petrucci MN, Motl RW, Hsiao-Wecksler ET.Six-Minute Walk Test Performance in Persons With Multiple Sclerosis While Using Passive or Powered Ankle-Foot Orthoses. Arch Phys Med Rehabil 2018; 99(3): 484-90.

3. Böing T. Ein Leitfaden zu Versorgungsoptionen bei Fußheberschwäche. Orthopädietechnik 2019; 70(3): 16-22.

4. Deems-Dluhy S, Hoppe-Ludwig S, Mummidisetty C, Lonini L, Shawen N, Jayaraman A. The Microprocessor Controlled Orthosis: What is the impact to the user versus the stance control orthosis and conventional locked KAFO? AOPA-Assembly 2017; 6. -9. September, Las Vegas, USA.

5. Deutsche Multiple Sklerose Gesellschaft 2019. Zugriff am 02. April 2020 unter https://www. dmsg.de/multiple-sklerosenews/ms-forschung/multiple-sklerose-drei-viertel-der-befragten-erkrankten-haben-einen-schubfoermigen-verlauf/

6. Deutsche Vereinigung für Rehabilitation e. V. 2017. Empfehlungen zur Verbesserung des teilhabeorientierten Versorgungsprozesses mobilitätseingeschränkter Menschen mit Rollstühlen. Zugriff am 31. März 2020 unter https://www.dvfr.de/ fileadmin/user_upload/DVfR/Downloads/Stellungnahmen/ DVfR-Stellungnahme_Rollstuhlversorgung_-_Juni_2018_bf.pdf

7. Flachenecker P, Kobelt G, Berg J, Capsa D, Gannedahl M. New insights into the burden and costs of multiple sclerosis in Europe: Results for Germany. Mult Scler 2017; 23(2): 78-90.

8. Flachenecker P, Zettl UK. Krankheitsverlauf und Prognose. In: Schmidt RM, Hoffmann F, Faiss JH, Köhler W. (Hrsg.) Multiple Sklerose, Urban \& Fischer/Elsevier 2015; 59-68.

9. GBD 2017 Disease and Injury Incidence and Prevalence Collaborators. Global, regional, and national disability-adjusted life-years (DALYs) for 359 diseases and injuries and healthy life expectancy (HALE) for 195 countries and territories, 19902017: a systematic analysis for the Global Burden of Disease Study 2017. Lancet 2018; 392: 1789-858.

10. GKV-Spitzenverband 2018. Entlassmanagement Rahmenvertrag. Zugriff am 02. April 2020 unter https://www.kbv.de/ media/sp/Rahmenvertrag_Entlassmanagement.pdf 
10a. GKV-Spitzenverband (2020). Kennzahlen der gesetzlichen Krankenversicherung. Zugriff am 02. April 2020 unter https:// www.gkv-spitzenverband.de/media/grafiken/gkv_kennzahlen/kennzahlen_gkv_2019_q4/GKV_Kennzahlen_Booklet_ Q4-2019_30odpi_2020-03-11.pdf

11. Glattacker M, Giesler JM, Klindtworth K, Nebe A. Rehabilitation use in multiple sclerosis: Do illness representations matter? Brain and behavior 2018; 8(6): eoo953. doi:10.1002/brb3.953

12. Halabchi F, Alizadeh Z, Sahraian MA, Abolhasani M. Exercise prescription for patients with multiple sclerosis; potential benefits and practical recommendations. BMC Neurology 2017; 17(1): 185. doi:10.1186/s12883-017-0960-9

13. Holstiege J, Steffen A, Goffrier B, Bätzing J. Epidemiologie der Multiplen Sklerose - eine populationsbasierte deutschlandweite Studie. Zentralinstitut für die kassenärztliche Versorgung in Deutschland (Zi). Versorgungsatlas-Bericht 2017; Nr. 17/09.

14. Kern C. Entwicklung eines therapeutischen Kletterprogramms und Evaluation seiner Effekte auf Personen mit Multipler Sklerose. Dissertation an der Fakultät für Sport- und Gesundheitswissenschaften, Technische Universität München 2014.

15. Kersten S, Mahli M, Haas C. Zum Sport- und Bewegungsverhalten von Multiple Sklerose Patienten - eine explorative Analyse. Aktuelle Neurologie 2014; 41(2): 100-6.

16. Khan F, Amatya B. Rehabilitation in multiple sclerosis: A systematic review of systematic reviews. Archives of PhysicalMedicine and Rehabilitation 2017; 98, 353-67.

17. Khurana SR, Beranger AG, Felix ER. Perceived Exertion Is Lower When Using a Functional Electrical Stimulation Neuroprosthesis Compared With an Ankle-Foot Orthosis in Persons With Multiple Sclerosis: A Preliminary Study. Am J Phys Med Rehabil 2017; 96(3): 133-9.

18. Kim Y, Lai B, Mehta T, Thirumalai M, Padalabalanarayanan S, Rimmer JH, Motl RW. Exercise Training Guidelines for Multiple Sclerosis, Stroke, and Parkinson Disease: Rapid Review and Synthesis. Am J Phys Med Rehabil 2019; 98(7): 613-21.

19. Kurtzke JF. Rating neurologic impairment in multiple sclerosis:ban expanded disability status scale (EDSS). Neurology 1983; 33: 1444-52.

20. Mattson E, Broström L. The increase in energy cost of walking with an immobilized knee or an unstable ankle. Scan J Rehab Med 1990; 22: 51-3.

21. Nowossadeck E. Einfluss der demografischen Alterung auf die Inanspruchnahme der medizinischen Rehabilitation in Deutschland bis 2040. Die Rehabilitation 2019; 58(02): 96-103.

22. Pröbsting E, Kannenberg A, Zacharias B. Safety and walking ability of KAFO users with the C-Brace ${ }^{\circledR}$ Orthotronic Mobility System, a new microprocessor stance and swing control orthosis. Prosthet Orthot Int 2017; 41(1): 65-77.

23. Richtlinie des Gemeinsamen Bundesausschusses über die Verordnung von Hilfsmitteln in der vertragsärztlichen Versorgung (2018). Zugriff am 26. Juli unter https://www.g-ba.de/downloads/62-492-1666/HilfsM-RL_2018-07-19_iK-2018-10-03.pdf

24. Rütten A, Pfeifer K. 2017 Nationale Empfehlungen für Bewegungund Bewegungsförderung. Zugriff am 11. Juni 2019 unter https://www.bundesgesundheitsministerium.de/fileadmin/ Dateien/5_Publikationen/Praevention/Broschueren/Bewegungsempfehlungen_BZgA-Fachheft_3.pdf

25. Sangelaji B, Kordi M, Banihashemi F, Nabavi SM, Khodadadeh S, Dastoorpoor M. A combined exercise model for improving muscle strength, balance, walking distance, and motor agility in multiple sclerosis patients: A randomized clinical trial. Iran J Neurol 2016; 15(3): 111-20.

26. Schmalz T, Blumentritt S, Drewitz H. Gangphasenabhängig entriegelnde versus gesperrte Beinorthesen - Biomechanische und metabolische Untersuchungen. Med Orth Tech 2005; 125(3): 67-74.

27. Stevens V, Goodman K, Rough K, Kraft GH. Gait impairment and optimizing mobility in multiple sclerosis. Phys Med Rehabil Clin N Am 2013; 24(4): 573-92.

28. Street T, Singleton C. Five-Year Follow-up of a Longitudinal Cohort Study of the Effectiveness of Functional Electrical Stimulation for People with Multiple Sclerosis. Int J MS Care 2018; 20: 224-30.
29. Street T, Taylor P, Swain I. Effectiveness of Functional Electrical Stimulation on Walking Speed, Functional Walking. Category, and Clinically Meaningful Changes for People With Multiple Sclerosis. Arch Phys Med Rehabil 2015; 96(4): 667-72.

30. Swinnen E, Deliens T, Dewulf E, Van Overstraeten S, Lefeber N, Van Nieuwenhoven J, Ilsbroukx S, Kerckhofs E. What is the opinion of patients with multiple sclerosis and their healthcare professionals about lower limb orthoses? A qualitative study using focus group discussions. NeuroRehabilitation 2018; 42(1): 81-92.

31. Tallner A, Waschbisch A, Wenny I, Schwab S, Hentschke C Pfeifer K, Mäurer M. Multiple sclerosis relapses are not associated with exercise. Multiple Sclerosis Journal 2012; 18(2): 232-5.

32. Tholen R, Dettmers C, Henze T, Höthker S, Flachenecker P, Lamprecht S, Sailer M, Tallner A, Vaney C. Bewegungstherapie zur Verbesserung der Mobilität von Patienten mit Multipler Sklerose. Konsensusfassung für die Sze-Leitlinie der DGNR in Zusammenarbeit mit Physio Deutschland - Deutscher Verband für Physiotherapie (ZVK) e. V. Neurol Rehabil 2018; 25(1): $03-40$.

33. Zacharias B, Kannenberg A. Clinical benefits of stance con trol orthosis systems: An analysis of the scientific literature. JProsth Orthot 2012; 24(1): 2-7.

34. Zheleznyakova GY, Piket E, Marabita F, Pahlevan Kakhki M, Ewing E, Ruhrmann S, Needhamsen M, Jagodic M, Kular M. Epigenetic research in multiple sclerosis: progress, challenges, and opportunities. Physiol Genomics 2017; 49(9): 447-61.

Interessenvermerk

Sabine Lamprecht gibt an, dass kein Interessenkonflikt besteht.

Dr. Thorsten Böing ist Leiter des Fachbereichs »Rehabilitation \& Versorgungsforschung « bei der Firma Otto Bock HealthCare Deutschland.

\section{Korrespondenzadresse:}

Sabine Lamprecht

HSH Lamprecht GbR

Otto-Ficker-Straße

73230 Kirchheim/Teck

slamprecht@hsh-lamprecht.de 